\title{
Análisis de las habilidades ortográficas en español del estudiantado universitario en Ecuador
}

Analysis of the orthographic skills in Spanish of Ecuadorian University students

\section{Volumen 21, Número 1 \\ Enero - Abril \\ pp. 1-21}

\author{
Deborah Valerie Montesdeoca Arteaga \\ Fátima Elizabeth Palacios Briones \\ María Elena Gómez-Parra \\ Roberto Espejo Mohedano
}

Citar este documento según modelo APA

Montesdeoca Arteaga, Deborah Valerie., Palacios Briones, Fátima Elizabeth., Gómez-Parra, María Elena. y Espejo Mohedano, Roberto. (2021). Análisis de las habilidades ortográficas en español del estudiantado universitario en Ecuador. Revista Actualidades Investigativas en Educación, 21(1), 1-21. Doi. 10.15517/aie.v21i1.44074 


\title{
Análisis de las habilidades ortográficas en español del estudiantado universitario en Ecuador
}

Analysis of the orthographic skills in Spanish of Ecuadorian University students

\author{
Deborah Valerie Montesdeoca Arteaga ${ }^{1}$ \\ Fátima Elizabeth Palacios Briones ${ }^{2}$ \\ María Elena Gómez-Parra ${ }^{3}$ \\ Roberto Espejo Mohedano 4
}

\begin{abstract}
Resumen: Las competencias ortográficas son destrezas esenciales del alumnado universitario, son parte del bagaje académico adquirido tanto en el nivel básico como en el nivel medio. No obstante, para el profesorado son un reto porque traen consigo deficiencias ortográficas cuando inician el nivel superior. Dada la situación descrita, el objetivo del estudio es diagnosticar la competencia ortográfica que posee el estudiantado universitario de primer semestre en las carreras de la Escuela Superior Politécnica Agropecuaria de Manabí Manuel Félix López (ESPAM MFL) en Ecuador. Se aplicó una evaluación diagnóstica a 438 asistentes matriculados durante las dos primeras semanas del período académico (Octubre 2015 - Marzo 2016), para el análisis de datos se utilizó la teoría descriptiva y tablas cruzadas, con lo cual se identificaron las falencias de carácter ortográfico y de vocabulario. Las variables demográficas utilizadas durante el análisis de los datos fueron el sexo, edad y la procedencia de estudios. Los resultados muestran que existe una carencia alarmante en la aplicación de las reglas ortográficas. Se observa la necesidad de desarrollar un programa para la mejora de la ortografía del alumnado universitario, por lo que se plantea el diseño de estrategias de solución a este problema ya que, una buena formación en ortografía es fundamental en el desarrollo personal y profesional del estudiantado universitario.
\end{abstract}

Palabras clave: ortografía, vocabulario, estudiante universitario, enseñanza superior.

Abstract: Spelling is an essential skill for university students as it is part of the academic background acquired at both basic and intermediate levels. However, it is a challenge for teachers because students bring spelling deficiencies with them when they start their higher education stage. Thus, the objective of the study is to diagnose the spelling competence of first-year university students in specific courses at Escuela Superior Politécnica Agropecuaria de Manabi Manuel Félix López (ESPAM MFL) in Ecuador. A diagnostic evaluation was applied to 438 students during the first two weeks of the academic period (October 2015 - March 2016). Descriptive theory and cross tables were used for data analysis, identifying spelling and vocabulary weaknesses. The demographic variables used during data analysis were sex, age and study origin. The results show that there is an alarming lack of application of spelling rules. The need to develop a program to improve the spelling of university students was identified. Therefore, strategies to solve this problem were designed, since good training in spelling is fundamental for the personal and professional development of university students.

Keywords: spelling, vocabulary, university students, higher education

1 Profesora de la Escuela Superior Politécnica Agropecuaria de Manabí Manuel Félix López, en la
Facultad de Educación, Calceta, Ecuador. Dirección electrónica:
deborah.montesdeoca@espam.edu.ec ORCID https://orcid.org/0000-0002-3662-5091

2 Profesora de la Escuela Superior Politécnica Agropecuaria de Manabí Manuel Félix López, en la Facultad de Educación, Calceta, Ecuador. Dirección electrónica: fapabri15@espam.edu.ec ORCID https://orcid.org/0000-0002-9662-08072

3 Profesora Titular de la Universidad de Córdoba, en la Facultad de Educación, Córdoba, España. Dirección electrónica elena.gomez@uco.es ORCID https://orcid.org/0000-0001-7870-35053

${ }^{4}$ Profesor de la Universidad de Córdoba, Dpto. de Estadística, Econometría, I.O. Organización de Empresas y Economía Aplicada. Córdoba, España. Dirección electrónica roberto.espejo@uco.es ORCID https://orcid.org/0000-0001-6592-35094

Artículo recibido: 16 de junio, 2020

Enviado a corrección: 3 de setiembre, 2020

Aprobado: 9 de noviembre, 2020

Los contenidos de este artículo están bajo una licencia Creative Commons 


\section{Introducción}

La escritura es una herramienta transversal para el buen desarrollo académico del estudiante universitario. Se trata de un instrumento con potencial epistémico (Carlino, 2005; Ruiz, 2009; Scardamalia y Beretir, 1992). Es una parte de la gramática y un aspecto importante de la expresión escrita, aunque no es el único ni el más significativo (Prado, 2016). No obstante, González, Rodríguez y Ledo (2019) la conciben como una actividad comunicativa que integra procesos mentales, lingüísticos y estilísticos complejos, en cuya interrelación descansa la efectividad de la comunicación en el código escrito. Así, es un parámetro importante a la hora de escribir, porque su correcta aplicación aporta legibilidad a un texto. Como parte importante de la escritura, la corrección ortográfica es indispensable para la expresión del hablante y, por tanto, debe constituirse en una competencia transversal en todas las materias universitarias. En la actualidad, el estudiantado muestra dificultades en el acceso a estudios superiores debido a la aparición de numerosas faltas de ortografía. Este hecho, por tanto, afecta su rendimiento académico y su desempeño profesional.

El alumnado que inicia los estudios universitarios debe mostrar competencias ortográficas suficientes, pero, como muestran los resultados de este estudio, existen importantes carencias en este sentido, lo que indica que el estudiantado arrastra falencias de los niveles que han precedido al nivel superior. Por eso, en lo que respecta a la enseñanza universitaria, es necesario que, junto a la enseñanza de los demás aspectos de la lengua escrita, se incluya el conocimiento ortográfico con especial incidencia en los vocablos, cuya escritura causa más problemas con el objeto de ayudar al alumnado a afianzar esta competencia. Es de señalar que tanto la comunicación oral como escrita responden a la necesidad de comunicarnos oralmente y por escrito de manera eficiente para interactuar en cualquier comunicación (Fonseca, Correa, Pineda y Lemus, 2011).

De acuerdo con la problemática existente, este estudio tiene por objetivo diagnosticar la competencia ortográfica que posee el alumnado universitario de primer semestre en las ocho carreras de la Escuela Superior Politécnica Agropecuaria de Manabí Manuel Félix López (ESPAM MFL) en Ecuador. Para la recolección de datos en este estudio, se tomó una muestra de 438 estudiantes del primer semestre de las carreras Administración Pública, Administración de Empresas, Agroindustria, Agrícola, Medicina Veterinaria, Computación, Turismo y Medio Ambiente de la universidad antes mencionada, durante las dos primeras semanas del período académico Octubre 2015 - Marzo 2016, lo que permitió un análisis completo de las habilidades ortográficas del alumnado que accede a cada una de estas carreras universitarias. Los 
resultados de este diagnóstico dan lugar al diseño de una propuesta de intervención para la mejora de la ortografía.

\section{Marco teórico}

\subsection{El término "ortografía"}

La palabra "ortografía" es de origen griego "ó $\rho \theta$ ", que significa "recto, correcto, derecho,

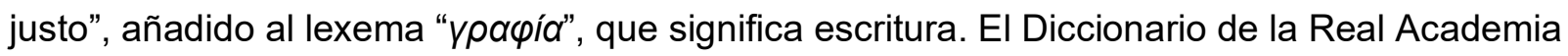
de la Lengua Española (1970, p. 741) citado por Camps, Milian, Bigas, Camps y Cabré (1990) la define como "parte de la gramática, que enseña a escribir correctamente por el acertado empleo de las letras y de los signos auxiliares de la escritura" (p. 7). En palabras de Escarpanter (1992), es el "estudio de la forma de representar, por medio de letras, los fonemas del lenguaje" (p.46), definición ratificada por Fernández- Rufete (2015) al afirmar que: "la ortografía está presente en cualquier actividad educativa y que, últimamente está adquiriendo más relevancia en la preocupación docente, porque, los escritos se encuentran plagados de multitud de faltas ortográficas" (p. 9) Ferreiro (1986) sostiene que:

La ortografía consiste en un sistema alfabético, sistema de grafemas, habitualmente llamados letras, que tienen una gran variedad de formas y estilos. La ortografía también incluye las reglas ortográficas y de puntuación, mediante las cuales, las letras pueden cambiarse para representar los sistemas fonéticos, morfofonémicos, morfémicos, sintácticos y pragmáticos del lenguaje. De tal manera, el alfabeto debe ser modificado ligeramente de lengua a lengua. Pero la ortografía también debe ser capaz de representar las maneras en que se combinan los sonidos del lenguaje y el modo en que son modificados por los sonidos circundantes. Al hacerlo, hay elecciones necesarias acerca del nivel del lenguaje que se va a representar, es decir, a veces se elige las palabras que parezcan semejantes, o bien porque están relacionadas al nivel gramatical o por el significado. La ortografía debe indicar a través de la puntuación las pautas de oración, frase y cláusula que representan significado. (p. 20)

Por tanto, existen motivos suficientemente justificados para analizar si existe una falencia de tipo ortográfico en el aula universitaria, así como para realizar propuestas de mejora, pues de una buena ortografía depende, en gran medida, que el mensaje llegue debidamente al receptor. 
Las diferentes formas de enseñar y de aprender ortografía en la escuela se basan en un patrón convencional de repetición y corrección de errores. Debido a la presencia de aspectos formales es necesario incluir elementos asociados a la legibilidad de textos en la evaluación de las materias universitarias. En este sentido, es importante distinguir problemas ortográficos que puedan afectar la legibilidad o la decodificación del texto escrito. Es de señalar que la ortografía es parte importante del conjunto del texto, y es por ello que debe evaluarse en su justa medida (Condemarín y Medina, 2000). En el currículo de enseñanza obligatoria, el aprendizaje de la lengua escrita se plantea de forma progresiva, apoyándose en un trabajo continuado sobre los procesos de comprensión y producción de textos escritos de creciente complejidad a lo largo de todo el trayecto educativo (Pérez y Zayas, 2007). Castro (2011) afirma:

La evaluación de competencias se puede definir como un procedimiento en el que se requiere que el estudiantado complete tareas o procesos en los que se demuestre su habilidad para aplicar conocimientos y destrezas o aplicar conocimientos en situaciones simuladas similares a la vida real. (p.118)

Cabe señalar que es importante realizar estudios que definan las habilidades ortográficas que aplica el alumnado en los modelos de la comunicación oral o escrita, ya que como indica García (2014), es necesario conocer cuáles son sus dificultades y establecer estrategias de aprendizaje apropiadas para superarlas y que, a la vez, conduzcan al estudiantado hacia el desarrollo de sus competencias académicas y profesionales con garantías de éxito. Además, al contexto comunicativo corresponde también la formación que tienen ambos interlocutores, y que el profesor debe interpretar para llegar de manera efectiva en el alumnado.

La dimensión ortográfica es una parte consustancial a cualquier proceso de formación en todas las disciplinas científicas. El trabajo coordinado del profesorado, en este sentido, puede resultar ciertamente significativo para trabajar la escritura académica y la ortografía desde todas las áreas del currículo universitario, lo cual debe dar resultados positivos.

Según la propuesta metodológica de Prado (2016), el interés tradicional de la ortografía se ha centrado en la relación entre grafía y fonema, postergando la importancia del acento y la puntuación. La jerarquía de los aspectos ortográficos, por orden de tratamiento pedagógico, ha sido la siguiente: el uso de las letras, el acento, la puntuación y otras convenciones gráficas, como las abreviaturas, correcta separación de palabras en sílabas, entre otros. La enseñanza 
integral, integrada y coordinada de la ortografía en los currículos universitarios ha de tener efectos positivos si esta se desarrolla junto con los demás aspectos de la lengua escrita, porque el fin es formar al estudiantado competitivo en la escritura académica, Por tanto, la enseñanza de conocimientos y habilidades se debe orientar de forma que contribuya a la formación integral del estudiantado. Toda actividad docente tiene que ser portadora de los objetivos educativos que se proponen en el modelo del profesional (Franco y Benites, 2018). Es por ello que su enseñanza y aprendizaje debe plantearse de forma sistemática en la Educación Superior.

\subsection{Causas de la deficiencia ortográfica}

La carencia de lectura es un punto donde convergen las perspectivas del profesorado y el alumnado como una de las causas fundamentales de la deficiente escritura académica. La literatura científica coincide en que la escasa importancia que se le da a la pulcritud de la expresión oral y escrita podría estar entre las causas más sobresalientes de la deficiente ortografía del alumnado universitario (Prado, 1998). Ortiz et al. (2009) indican que

Es fundamental en el proceso de aprendizaje de la lengua escrita, la creación de un entorno alfabetizador donde se promueva el gusto y valoración por la lectura y la escritura, lo que llevará al estudiante a tener conceptos y sentimientos positivos hacia ellas y desarrollar, por ende, sus intenciones de leer y escribir. (p. 96)

En este aspecto, Carlino (2013) sostiene que es necesario que se impartan talleres de redacción al inicio de los semestres. Sin embargo, suelen resultar insuficientes porque se aprenden solo las generalidades para escribir y leer. Por ende, es necesario adquirir habilidades de lectoescritura en cada materia y área del conocimiento. La escritura académica es vista por el estudiantado como una actividad propia del profesorado e investigadores, y no como su responsabilidad en aprender a utilizarla, porque consideran que corresponde a esferas elitistas de las universidades, deficiencia que también es observada en los docentes cuando explican los temas de clases y demuestran falta de conocimiento de estrategias para redactar, falta de atención en clases relacionadas con lengua y literatura en niveles anteriores, falta de información, práctica y asesoría ${ }^{5}$ (Hermosillo y Verdín, 2019).

\footnotetext{
${ }^{5}$ Conclusión de un estudio en torno a la escritura académica realizado a estudiantes y docentes de una Universidad Privada de la Zona Metropolitana de Guadalajara, México.
} 
Además, el alumnado concibe la escritura académica como un medio para hacer tareas, cumplir con lo solicitado en sus asignaturas y obtener un título profesional, lo cual corresponde a los discursos producidos con respecto a esta práctica en su contexto familiar, laboral, entre otros (Van Dijk, 2010). Otra recurrencia en las opiniones del profesorado es que los trabajos académicos realizados por el alumnado carecen de dirección y difusión, por lo que no se les brinda la "oportunidad profesional de demostrar sus competencias comunicativas que pongan en juego el uso de la escritura" (Hermosillo y Verdín, 2019, p. 48).

En este sentido, se determina que las estrategias aplicadas por el profesorado en la construcción de las tareas académicas permiten que el estudiantado disponga de herramientas que fundamenten la producción de textos escritos y que, al mismo tiempo, genere competencias comunicativas durante la etapa profesional. "Los textos escritos que produce el estudiantado son para el profesorado, para ser leídos y evaluados por él, no son leídos en clase, ni son compartidos entre los compañeros" (Serrano de Moreno, Duque de Duque y Madrid de Forero, 2012, p. 104).

Los factores señalados inciden en el uso de una ortografía deficiente. También, se considera que la lectura es fundamental, a pesar de que ha perdido protagonismo dentro de los hábitos del estudiantado, como sostiene Sánchez (2009) cuando plantea que la lectura es un óptimo auxiliar de la ortografía porque contribuye a reforzar la imagen léxica de las palabras, afianzando el léxico que el usuario ya conoce.

En los Grados Universitarios, en Ecuador, el alumnado muestra dificultades en el área de escritura. Según Guzmán y Moya (2016) principalmente, durante los primeros ciclos de estudio se observa serias dificultades en el área de la escritura, principalmente en la ortografía, lo cual es producto de la falta de aprehensión de las reglas y de una instrucción deficiente.

Se suma a ello el problema de actitud, puesto que el estudiantado no muestra interés especial en escribir sus textos sin faltas de ortografía. Por eso, es necesaria una profunda reflexión y análisis de la importancia del dominio de la competencia escrita (Gómez Camacho, 2013).

\subsection{Importancia de la ortografía y su vínculo con la comunicación escrita}

Las autoras Errázuriz y Fuentes (2012) indican que el alumnado de primer año de la carrera de Pedagogía General Básica (carrera de formación docente destinada a impartir clases de primer a octavo grado) ingresa con una puntuación inferior a 600 puntos en la Prueba de Selección Universitaria en Chile (PSU), y que sus promedios de lenguaje son aún más bajos 
que el promedio ponderado, lo que evidencia que las habilidades comunicativas son bajas, ocasionando un déficit en el proceso de aprendizaje general.

En este mismo contexto, varios estudios (Flotts et al., 2015; Flotts et al., 2016; Vallejo, Vidal, Rivadeneira, Benavídez y Raza, 2008) revelan resultados similares en las pruebas SER ECUADOR $^{6}$, TERCE ${ }^{7}$ y SER BACHILLER ${ }^{8}$, aplicadas en quince países de América Latina (dentro de los cuales está Ecuador), y cuya puntuación promedio en Lenguaje y Comunicación se estableció en 500 puntos (para las primeras pruebas). Solo 9 provincias de las 24 que integran el Ecuador superan el promedio en este campo, los 15 restantes se encuentran por debajo del promedio, incluyendo al estudiantado de Manabí que obtuvieron una puntuación de 485 en cuarto año de Educación General Básica (EGB), y una puntuación de 467, los de séptimo grado. Es notable la tendencia a la baja a medida que van incrementando los años de estudio. Es de señalar que los resultados del estudio de 2012 , en el alumnado ecuatoriano de enseñanza general básica y bachillerato, no han sido publicados formalmente por el Instituto Nacional de Evaluación (INEVAL), entidad responsable del proceso de evaluación en el país.

Al respecto, Flotts et al., (2015) replican que se evidenciaron un alto porcentaje de deficiencia en comprensión lectora y una media significativamente inferior en escritura en relación con el resto de los países evaluados para estudiantes de Educación General Básica. Estos resultados son corroborados por las pruebas SER BACHILLER, que el INEVAL presentó en el año 2016, en las cuales, a nivel del Ecuador, en el área de Lengua y Literatura, los bachilleres presentan una puntuación de 786/1000 (es decir, equivalente a nivel elemental). Si bien es cierto se ha elevado el resultado en relación con las pruebas de 2008 , no lo es menos que el nivel de excelencia es menor que insuficiente.

\footnotetext{
6 Por primera vez se aplicó estas pruebas en el año 2008 de manera censal a estudiantes de establecimientos educativos fiscales, fiscomisionales, municipales y particulares, en los siguientes años: cuarto, séptimo y décimo de Educación Básica, y tercero de Bachillerato y en las áreas de Matemática y Lenguaje y Comunicación.

7 Tercer Estudio Regional Comparativo y Explicativo, que analiza el logro de aprendizaje a gran escala más importante de la región, ya que comprende 15 países (Argentina, Brasil, Chile, Colombia, Costa Rica, Ecuador, Guatemala, Honduras, México, Nicaragua, Panamá, Paraguay, Perú, República Dominicana y Uruguay) más el Estado de Nuevo León (México). Este estudio evalúa el desempeño escolar en tercer y sexto grados de escuela primaria en las áreas de Matemática, Lenguaje (lectura y escritura) y, para sexto grado el área de Ciencias Naturales. Su objetivo principal es aportar información para el debate sobre la calidad de la educación en la región, así como orientar la toma de decisiones en políticas públicas educativas.

8 Pruebas dirigidas al alumnado de tercero de bachillerato en modalidad presencial, semipresencial y a distancia de Ecuador que hayan aprobado las asignaturas del currículo de tercero de Bachillerato. La prueba reemplaza a los exámenes de grado y evalúa cuatro campos: Matemática, Lengua y Literatura, Ciencias Naturales y Estudios Sociales, que están determinados en los Estándares de Calidad Educativa del Mineduc (Ministerio de Educación).
} 
Tomando como referente estos resultados, y a partir de la información proveniente del análisis curricular del $\mathrm{SERCE}^{9}$ y la actualización para el TERCE, queda de manifiesto el interés de los países latinoamericanos por la corrección en la escritura. En la misma línea, Flotts et al., (2016) citan que, en la enseñanza de la escritura, se estarían descuidando elementos de escritura interna o dimensión construccional del texto, como: coherencia global, concordancia y cohesión. Además, Dido (2001) señala que la escuela secundaria remite el problema a la primaria, al tiempo que la universidad denuncia la situación y se declara impotente, clamando por soluciones en los niveles inferiores sobre los serios problemas ortográficos y de puntuación en la escritura. Esto indicaría que un semestre de dos horas no es suficiente para subsanar los vacíos que trae el alumnado de la escolaridad anterior (Cisneros, Muñoz y Herrera, 2014, p. 186).

Kaufman (2017) sostiene:

Saber escribir implica tener claros los propósitos que orientan mi accionar, saber quién es el destinatario, conocer las características del tipo de texto que se desea producir, utilizar correctamente las variadas estrategias de cohesión y coherencia textual, emplear los recursos retóricos al servicio de la intencionalidad del autor, tener el conocimiento gramatical necesario para poder articular la sintaxis de la manera más conveniente $y$, también, respetar las convenciones ortográficas vinculadas con la escritura de las palabras y el uso de la puntuación a fin de que el texto sea eficaz. (p. 24)

Es decir, durante el proceso de escritura los alumnos no solo aprenden a escribir en una lengua, sino que perfeccionan las otras destrezas comunicativas al intercambiar y compartir ideas, puesto que, aprender a escribir significa aprender a organizar ideas, construir textos con coherencia lógica, adaptar el estilo según el destinatario, el tema tratado y el tipo de texto (Palma, 2012, p. 34). Por lo que, Gómez (1998) indica tres razones por las que es importante saber expresarse adecuadamente por escrito:

a) Cuanto más se domine la expresión escrita, mayor será la posibilidad de convencer y obtener una respuesta;

\footnotetext{
9 Segundo Estudio Regional Comparativo y Explicativo, evaluó el logro de aprendizaje de 100.752 estudiantes de tercer grado y 95.288 de sexto grado de 16 países más el Estado mexicano de Nuevo León, en matemática, lectura y escritura y ciencias de la naturaleza constituyendo, entonces, el estudio de calidad de la educación más grande implementado en América Latina y el Caribe hasta esa fecha. El estudio además indagó sobre los factores escolares y sociales que se asocian y posiblemente explican el logro del estudiantado.
} 
b) La mayor parte de las evaluaciones académicas que realiza el alumnado son ejercicios escritos, y;

c) A través de la escritura se expresan sentimientos y emociones.

Para mejorar esta competencia, por tanto, es necesario un cambio conceptual tanto en el profesorado como en el estudiantado, que permita implementar acciones tendentes a resolver los problemas diagnosticados en este estudio. Asimismo, sería de gran importancia que estas acciones también se dirigiesen a los docentes universitarios, ya que son ellos los responsables de iniciar una labor de alfabetización y escritura académica correcta en Educación Superior (Carlino, 2005).

\subsection{La escritura a través del currículo en Educación Superior}

Entre los factores que se vinculan al éxito del alumnado en la universidad destacan dos: la capacidad de aprender y la de utilizar las prácticas de comunicación oral y escrita tipificadas por la comunidad de aprendizaje (Pérez, Navarro y Waigandt, 2017). Se trata, pues de herramientas privilegiadas (Castelló, 2009; Thaiss, y Zawacki, 2006) que garantizan en gran parte el objetivo del proceso de aprendizaje. Leer, escribir y comunicarse oralmente son habilidades claves para el desempeño del estudiantado en dos momentos clave: durante su formación en Educación Superior y en el desarrollo de su profesión.

La presencia de la asignatura de Lenguaje y Comunicación en los currículos universitarios de primer semestre, en casi la totalidad de las carreras universitarias de Ecuador, aparecen con la necesidad de que el alumnado fortalezca la lectura y escritura, ya que, cuya percepción es crítica en el país, porque la variabilidad en el análisis de escritura del estudio TERCE se ubica en 1,33 puntos en comparación con los demás países evaluados (Flotts et al., 2016, p.103).

Por tanto, identificar la existencia de dificultades en este sentido, analizar los resultados y realizar una propuesta de mejora redunda en una mejor calidad de expresión académica para el alumnado universitario. 


\section{Metodología}

\subsection{Enfoque}

El enfoque de esta investigación es de tipo cuantitativo, teniendo un alcance descriptivo y correlacional. Esta investigación tuvo como objetivo principal diagnosticar la competencia ortográfica y de vocabulario que posee el estudiantado universitario de primer semestre en carreras de la Escuela Superior Politécnica Agropecuaria de Manabí "Manuel Félix López" (ESPAM MFL).

Para su desarrollo, se plantearon las siguientes hipótesis:

$\mathrm{H} 1$. Existen altos niveles de faltas ortográficas en el estudiantado universitario de primer semestre en las carreras de la ESPAM MFL.

$\mathrm{H} 2$. El nivel de vocabulario en el estudiantado universitario de primer semestre en las carreras de la ESPAM MFL es deficiente.

H3. Tanto el nivel ortográfico en el alumnado de primer semestre en las carreras de la ESPAM MFL como el de vocabulario depende de la titulación en la que estén enrolados.

$\mathrm{H} 4$. Existe una correlación entre las falencias de la dimensión ortográfica y el vocabulario en el alumnado universitario de primer semestre en las carreras de la ESPAM MFL.

Para la consecución del objetivo principal, se platearon las siguientes estrategias:

a) Aplicar una prueba que evalúe la competencia ortográfica y otra de vocabulario en el estudiantado universitario de primer semestre en las carreras de la ESPAM MFL.

b) Realizar un análisis descriptivo comparativo entre ortografía y vocabulario, como componentes de la producción escrita, en el estudiantado universitario de primer semestre en las carreras de la ESPAM MFL.

c) Aplicar test de independencia de la familia chi-cuadrado para corroborar si las falencias en las dimensiones ortográficas y de vocabulario dependen de la titulación en la que se encuentren enrolados el estudiantado universitario de primer semestre en las carreras de la ESPAM MFL.

d) Analizar las correlaciones existentes entre los niveles de ortografía y vocabulario, mediante el test de correlación de Spearman, en el estudiantado de primer semestre en las carreras de la ESPAM MFL. 
El análisis de los resultados se llevó a cabo mediante los procedimientos descriptivos y Tablas cruzadas del software estadístico SPSS V.22. El nivel de significación en los test estadísticos usados para aceptar o rechazar las hipótesis planteadas es del $5 \%$, es decir un a $=0,05$.

\subsection{Instrumento}

Para alcanzar los objetivos propuestos se diseñó un instrumento ad hoc, tomando como referencia las aportaciones de autores como Namorado (1995). El instrumento inicial estuvo compuesto por dos secciones; la primera sección contiene datos de tipo sociodemográfico (edad, sexo, estado civil, carrera que cursa, religión y sostenimiento de la institución) y la segunda sección está formada por dos dimensiones (ortografía y vocabulario). Para este análisis se consideró la dimensión ortografía, que consistió en identificar 10 errores o faltas de acentuación incorrecta, supresión y alteración de letras en un texto de 4 líneas (78 palabras). El nivel ortográfico del alumnado se midió a través de la siguiente escala de aciertos: excelente (10), muy bueno (9-8), bueno (7-6), regular (4-5), deficiente (3 o menos).

\subsection{Unidades de análisis y técnicas de recolección}

La prueba con el alumnado se la realizó por conveniencia en la ESPAM MFL durante las dos primeras semanas del período académico Octubre 2015 - Marzo 2016. Se tomó como base de estudio toda la población del alumnado de primer semestre. La población completa estaba constituida por 545 individuos a los que se les suministraron las pruebas de diagnóstico y que respondieron de forma voluntaria. El índice de respuesta fue muy elevado, 438 participantes que representan el $80,37 \%$ del total, lo que permitió disponer de información suficiente para describir la realidad del estudiantado que comienza sus estudios en la ESPAM MLF en lo referente a competencias ortográficas y de vocabulario (ver Tabla 1).

Las variables sociodemográficas utilizadas durante el análisis de los datos fueron el sexo (223 hombres y 215 mujeres), edad, y carrera universitaria en estudio. La edad media de los participantes fue de 19 años, siendo el $51 \%$ hombres y el $49 \%$ mujeres. 
Tabla 1

Ecuador: Población y muestra del estudiantado de primer semestre de las carreras universitarias de la ESPAM-MFL, periodo Octubre 2015 - Marzo 2016. (Valores nominales)

\begin{tabular}{|c|c|c|c|c|}
\hline \multirow[b]{2}{*}{ Carreras } & \multirow[b]{2}{*}{ Población } & \multicolumn{3}{|c|}{ Muestra } \\
\hline & & Hombre & Mujer & Total \\
\hline Administración de Empresas & 40 & 11 & 24 & 35 \\
\hline Administración Pública & 39 & 15 & 22 & 37 \\
\hline Agrícola & 91 & 40 & 25 & 65 \\
\hline Agroindustria & 89 & 37 & 40 & 77 \\
\hline Computación & 46 & 22 & 12 & 34 \\
\hline Medio Ambiente & 103 & 44 & 28 & 72 \\
\hline Medicina Veterinaria & 75 & 34 & 32 & 66 \\
\hline Turismo & 62 & 20 & 32 & 52 \\
\hline Total & 545 & 223 & 215 & 438 \\
\hline
\end{tabular}

Fuente: Elaboración propia con información de secretaría de área de la ESPAM-MFL de Ecuador, 2015

\section{Resultados}

En la Tabla 2, se presenta el porcentaje de los 438 estudiantes en la evaluación de ortografía distribuida según sus carreras universitarias. Como puede observarse, solo el 1,4 \% del estudiantado presenta un nivel de ortografía excelente, frente al 21,9\% clasificado como ortográficamente deficientes.

Los resultados que arroja la Tabla 2 permiten comparar los resultados del alumnado de acuerdo con las carreras universitarias en las que se matricularon. Cabe señalar que el alumnado de las titulaciones de Administración de Empresas, Administración Pública, Computación y Medicina Veterinaria no han alcanzado la calificación de excelente, lo que constituye un valor que es tenido en cuenta por las autoridades académicas. En cuanto al porcentaje del alumnado en las carreras de Computación (35,3\%), Turismo $(34,5 \%)$ y Agrícola (32,3 \%) presentan alto grado de deficiencia en ortografía, arrojando nuevamente un resultado alarmante. Es de señalar también que solo el alumnado de las titulaciones de Administración Pública y Medio Ambiente superan el 50 \% de los calificados como bueno o superior.

Estas diferencias entre titulaciones hacen sospechar que el nivel ortográfico del alumnado depende de la titulación en la que estén enrolados. Para responder a esta pregunta se realizó un test estadístico de independencia, de la familia chi-cuadrado, entre estas dos variables: carrera en estudio y nivel ortográfico. 
Tabla 2

Ecuador: Datos porcentuales de la prueba ortográfica en el estudiantado de primer semestre de las carreras universitarias de la ESPAM-MFL, periodo Octubre 2015 - Marzo 2016.

\begin{tabular}{|c|c|c|c|c|c|}
\hline \multicolumn{6}{|c|}{ Ortogratıa [0] } \\
\hline Carrera & $\begin{array}{c}\text { Deficiente } \\
(\leq 3)\end{array}$ & $\begin{array}{l}\text { Regular } \\
(\geq 4 \text { y }<6)\end{array}$ & $\begin{array}{c}\text { Bueno } \\
(\geq 6 \text { y }<8)\end{array}$ & $\begin{array}{c}\text { Muy bueno } \\
(\geq 8 \text { y }<9)\end{array}$ & $\begin{array}{c}\text { Excelente } \\
(\geq 9)\end{array}$ \\
\hline Administración de Empresas & 8,6 & $51,4 \%$ & $31,4 \%$ & $8,6 \%$ & $0,00 \%$ \\
\hline Administración Pública & $5,4 \%$ & $21,6 \%$ & $59,5 \%$ & $13,5 \%$ & $0,00 \%$ \\
\hline Agrícola & $32,3 \%$ & $38,5 \%$ & $24,6 \%$ & $3,1 \%$ & $1,5 \%$ \\
\hline Agroindustria & $27,3 \%$ & $28,6 \%$ & $35,0 \%$ & $7,8 \%$ & $1,3 \%$ \\
\hline Computación & $35,3 \%$ & $17,7 \%$ & $23,5 \%$ & $23,5 \%$ & $0,00 \%$ \\
\hline Medio Ambiente & $9,7 \%$ & $16,7 \%$ & $51,4 \%$ & $19,4 \%$ & $2,8 \%$ \\
\hline Medicina Veterinaria & $18,2 \%$ & $40,9 \%$ & $34,8 \%$ & $6,1 \%$ & $0,00 \%$ \\
\hline Turismo & $34,5 \%$ & $27,0 \%$ & $17,3 \%$ & $17,3 \%$ & $3,9 \%$ \\
\hline Resultado general & $21,9 \%$ & $30,2 \%$ & $34,9 \%$ & $11,6 \%$ & $1,4 \%$ \\
\hline
\end{tabular}

Fuente: Elaboración propia.

Para ello, y puesto que en la tabla de contingencia existen frecuencias con valor cero, se han agregado las categorías deficiente y regular en una sola, y las categorías muy bueno y excelente en otra. Los resultados porcentuales de esta agregación se muestran en la Tabla 3 y los resultados del test estadístico en la Tabla 4.

Tabla 3

Ecuador: Datos porcentuales agregados de la prueba ortográfica en el estudiantado de primer semestre de las carreras universitarias de la ESPAM-MFL, periodo Octubre 2015 - Marzo 2016. Ortografía [O]

\begin{tabular}{lccc}
\hline \multicolumn{1}{c}{ Carrera } & $\begin{array}{c}\text { Deficiente } \\
\text { regular }(<6)\end{array}$ & $\begin{array}{c}\text { Bueno } \\
(\geq \mathbf{6} \mathbf{~}<\mathbf{8})\end{array}$ & $\begin{array}{c}\text { Muy bueno o } \\
\text { excelente }(\geq \mathbf{8})\end{array}$ \\
\hline Administración de Empresas & $60,0 \%$ & $31,4 \%$ & $8,6 \%$ \\
Administración Pública & $27,0 \%$ & $59,5 \%$ & $13,5 \%$ \\
Agrícola & $70,8 \%$ & $24,6 \%$ & $4,6 \%$ \\
Agroindustria & $55,8 \%$ & $35,0 \%$ & $9,2 \%$ \\
Computación & $53,0 \%$ & $23,5 \%$ & $23,5 \%$ \\
Medio Ambiente & $26,4 \%$ & $51,4 \%$ & $22,2 \%$ \\
Medicina Veterinaria & $59,1 \%$ & $34,8 \%$ & $6,1 \%$ \\
Turismo & $61,5 \%$ & $17,3 \%$ & $21,2 \%$ \\
\hline \multicolumn{1}{c}{ Resultado general } & $\mathbf{5 2 , 1 \%}$ & $\mathbf{3 4 , 9} \%$ & $\mathbf{1 3 , 0} \%$ \\
\hline
\end{tabular}

Fuente: Elaboración propia.

Como puede observarse en la Tabla 4, tanto los valores del coeficiente Chi-cuadrado de Pearson, razón de verosimilitud, como de la $\mathrm{V}$ de Cramer son estadísticamente significativos ya que, en los tres casos, presentan un p-valor por debajo del nivel de significación establecido de $\alpha=0,05$, lo que infiere una relación entre ambas variables, como se sospechaba. 
Tabla 4

Ecuador: Prueba de independencia: ortografía en el estudiantado de primer semestre de las carreras universitarias de la ESPAM-MFL, periodo Octubre 2015 - Marzo 2016.

Chi-cuadrado de Pearson

Razón de verosimilitud

$\checkmark$ de Cramer

N

438

438

438
Valor

57,704

59,930

0,257

g.I

14

14 p-valor $<0,0001$

$<0,0001$ $<0,0001$

Fuente: Elaboración propia. 3 casillas $(12,5 \%)$ han esperado un recuento menor que 5.

El recuento mínimo esperado es 4,42 .

Centrándose ahora en los resultados que arroja la dimensión de vocabulario (ver Tabla 5), estos informan sobre problemas del alumnado en escritura, pues los datos marcados como "deficientes y regulares" son del orden del 57,8 \%, mientras que entre los "muy buenos y la excelencia" del 10,5\%.

Puede observarse también en la Tabla 5 que en lo que se refiere a vocabulario, la carrera de Medicina Veterinaria es la que tiene mayor cantidad de "insuficientes" y "regulares" (47,0 \%). Cabe destacar también el hecho de que muy pocos estudiantes alcanzan la excelencia en vocabulario. En particular, en las titulaciones de Agroindustria y Medicina Veterinaria no tienen estudiantado que alcance el nivel muy bueno o de excelencia. Esta situación nos alerta de nuevo de graves carencias en referencia al vocabulario de los alumnos Universitarios en la ESPAM MFL.

Tabla 5

Ecuador: Datos porcentuales de la prueba de vocabulario en estudiantes de primer semestre de las carreras universitarias de la ESPAM-MFL, periodo Octubre 2015 - Marzo 2016. Vocabulario [V]

\begin{tabular}{lccccc}
\hline arrera & $\begin{array}{c}\text { Deficiente } \\
(\leq 3)\end{array}$ & $\begin{array}{c}\text { Regular }(\geq \mathbf{4} \\
\mathbf{y}<\mathbf{6})\end{array}$ & $\begin{array}{c}\text { Bueno } \\
(\mathbf{2} \mathbf{6} \mathbf{y}<\mathbf{8})\end{array}$ & $\begin{array}{c}\text { Muy bueno } \\
(\mathbf{8} \mathbf{y}<\mathbf{9})\end{array}$ & $\begin{array}{c}\text { Excelente } \\
(\geq \mathbf{9})\end{array}$ \\
\hline Administración de Empresas & $0,00 \%$ & $31,4 \%$ & $65,7 \%$ & $2,9 \%$ & $0,00 \%$ \\
Administración Pública & $0,00 \%$ & $16,2 \%$ & $37,8 \%$ & $40,6 \%$ & $5,4 \%$ \\
Agrícola & $21,5 \%$ & $57,0 \%$ & $21,5 \%$ & $0,00 \%$ & $0,00 \%$ \\
Agroindustria & $6,5 \%$ & $41,6 \%$ & $36,4 \%$ & $15,5 \%$ & $0,00 \%$ \\
Computación & $14,7 \%$ & $64,7 \%$ & $17,6 \%$ & $3,0 \%$ & $0,00 \%$ \\
Medio Ambiente & $5,6 \%$ & $32,0 \%$ & $43,1 \%$ & $13,9 \%$ & $5,6 \%$ \\
Medicina Veterinaria & $47,0 \%$ & $47,0 \%$ & $6,0 \%$ & $0,00 \%$ & $0,00 \%$ \\
Turismo & $11,5 \%$ & $50,00 \%$ & $36,5 \%$ & $2,0 \%$ & $0,00 \%$ \\
\hline Resultado general & $\mathbf{1 4 , 9} \%$ & $\mathbf{4 2 , 9} \%$ & $\mathbf{3 1 , 7 \%}$ & $\mathbf{9 , 1} \%$ & $\mathbf{1 , 4} \%$ \\
\hline
\end{tabular}

Fuente: Elaboración propia.

En vista de los resultados mostrados, también cabría pensar que las puntuaciones obtenidas en vocabulario dependen de la titulación que se esté estudiando. Para corroborar esta sospecha, se procedió a aplicar un test de independencia entre las variables carrera en estudio y nivel de vocabulario. Como ocurría en el caso de la ortografía, existen celdas en la 
Tabla de contingencia con valor nulo, con lo que antes de realizar los test correspondientes se ha procedido a una agregación de categorías, en este caso considerando como una sola categoría la formada por deficiente y regular y, por otro lado, las formadas por bueno o superior. Los resultados porcentuales de la agregación se muestran en la Tabla 6.

Tabla 6

Ecuador: Datos porcentuales agregados de la prueba de vocabulario en el estudiantado de primer semestre de las carreras universitarias de la ESPAM-MFL, periodo Octubre 2015 - Marzo 2016.

Ortografía [V]

\begin{tabular}{lcc}
\hline \multicolumn{1}{c}{ Carrera } & $\begin{array}{c}\text { Deficiente o regular } \\
(<6)\end{array}$ & $\begin{array}{c}\text { Bueno o superior } \\
(\geq 6 \mathbf{~ y < 8 )}\end{array}$ \\
\hline Administración de Empresas & $31,4 \%$ & $68,6 \%$ \\
Administración Pública & $16,2 \%$ & $83,8 \%$ \\
Agrícola & $78,5 \%$ & $21,5 \%$ \\
Agroindustria & $48,1 \%$ & $51,9 \%$ \\
Computación & $79,4 \%$ & $20,6 \%$ \\
Medio Ambiente & $37,5 \%$ & $62,5 \%$ \\
Medicina Veterinaria & $93,9 \%$ & $6,1 \%$ \\
Turismo & $61,5 \%$ & $38,5 \%$ \\
\hline Resultado general & $\mathbf{5 2 7 , 8} \%$ & $\mathbf{4 2 , 2} \%$ \\
\hline
\end{tabular}

Fuente: Elaboración propia.

En la Tabla 7 se proporcionan los resultados de aplicar los test de independencia, y como puede observarse (en los tres casos el $p$-valor es prácticamente nulo y por menor que $\alpha=$ $0,05)$, también puede afirmarse la existencia de una relación estadísticamente significativa entre el nivel de vocabulario y la carrera en estudio.

Tabla 7

Ecuador: Prueba Chi-cuadrado de Pearson de independencia: vocabulario en el estudiantado de primer semestre de las carreras universitarias de la ESPAM-MFL, periodo Octubre 2015 Marzo 2016.

\begin{tabular}{lcccc}
\hline & $\mathbf{N}$ & Valor & g.l & p-valor \\
\hline Chi-cuadrado de Pearson & 438 & 104,873 & 7 & $<0,0001$ \\
Razón de verosimilitud & 438 & 116,553 & 7 & $<0,0001$ \\
V de Cramer & 438 & 0,489 & & $<0,0001$ \\
\hline
\end{tabular}

Fuente: Elaboración propia. 0 casillas $(0,0 \%)$ han esperado un recuento menor que 5.

El recuento mínimo esperado es 14,36 .

Según Murillo (2006), “lograr que el alumno escriba con ortografía requiere un poco más que buenas intenciones; es necesario conocer cuál es el vocabulario básico que usa al escribir, cuáles son las palabras que producen mayor número de errores" (p. 60). 
Por lo tanto, se analizó la existencia de relación entre las calificaciones obtenidas en las pruebas de ortografía y vocabulario. Para ello, usamos los coeficientes de correlación para variables de tipo ordinal de Spearman y coeficiente gamma. Los valores son mostrados en la Tabla 8, y ratifican la relación entre estas dos variables ( $p$-valor $<\alpha=0,05$ ), es decir, aquel alumnado que presenta problemas de ortografía también los tienen en vocabulario y viceversa.

Tabla 8

Ecuador: Prueba de independencia: vocabulario - ortografía en el estudiantado de primer semestre de las carreras universitarias de la ESPAM-MFL, periodo Octubre 2015 - Marzo 2016.

\begin{tabular}{lcccc}
\hline & $\mathbf{N}$ & Valor & Error estándar & p-valor \\
\hline Correlación de Spearman & 438 &, 276 & 0,045 & $<0,0001$ \\
Gamma & 438 &, 330 & 0,053 & $<0,0001$ \\
\hline
\end{tabular}

Fuente: Elaboración propia.

Sin embargo, si particularizamos este análisis por carreras de estudio, los resultados muestran que estás relaciones dependen de la carrera que estudian. Solo en las titulaciones de Administración de Empresas y Agroindustria aceptaríamos relación entre las calificaciones de ortografía y vocabulario ( $p$-valor $<\alpha=0,05$ ). En el resto de las titulaciones, el número de fallos (o aciertos) en las pruebas de ortografía y vocabulario no están correlacionados (ver Tabla 9).

Tabla 9

Ecuador: Prueba de independencia: vocabulario - ortografía en el estudiantado de primer semestre de las carreras universitarias de la ESPAM-MFL, periodo Octubre 2015 - Marzo 2016.

Vocabulario-Ortografía [VO]

\begin{tabular}{|c|c|c|c|c|c|}
\hline Carrera & & $\mathbf{N}$ & Valor & $\begin{array}{c}\text { Error } \\
\text { estándar }\end{array}$ & p-valor \\
\hline Administración & Correlación de Spearman & 35 & 0,402 & 0,143 & 0,017 \\
\hline Empresas & Gamma & & 0,670 & 0,198 & 0,012 \\
\hline Administración & Correlación de Spearman & 37 & $-0,023$ & 0,189 & 0,893 \\
\hline Pública & Gamma & & $-0,035$ & 0,251 & 0,887 \\
\hline \multirow[t]{2}{*}{ Agrícola } & Correlación de Spearman & 65 & 0,091 & 0,096 & 0,470 \\
\hline & Gamma & & 0,118 & 0,124 & 0,344 \\
\hline \multirow[t]{2}{*}{ Agroindustria } & Correlación de Spearman & 77 & 0,401 & 0,114 & 0,001 \\
\hline & Gamma & & 0,490 & 0,132 & 0,001 \\
\hline \multirow[t]{2}{*}{ Computación } & Correlación de Spearman & 34 &,- 009 & 0,193 & 0,961 \\
\hline & Gamma & &,- 004 & 0,277 & 0,987 \\
\hline \multirow[t]{2}{*}{ Medio Ambiente } & Correlación de Spearman & 72 & 0,188 & 0,120 & 0,113 \\
\hline & Gamma & & 0,233 & 0,148 & 0,125 \\
\hline \multirow[t]{2}{*}{ Medicina Veterinaria } & Correlación de Spearman & 66 & 0,044 & 0,120 & 0,727 \\
\hline & Gamma & 52 & 0,066 & 0,178 & 0,710 \\
\hline \multirow[t]{2}{*}{ Turismo } & Correlación de Spearman & & 0,192 & 0,135 & 0,173 \\
\hline & Gamma & & 0,241 & 0,169 & 0,157 \\
\hline
\end{tabular}

Fuente: Elaboración propia. 


\section{Conclusiones}

El objetivo principal de este estudio consistió en diagnosticar la competencia ortográfica y de vocabulario que posee el estudiantado de primer semestre en las carreras de la ESPAM MFL. Los resultados de la hipótesis permitieron evidenciar que las carreras de Computación, Turismo, Agrícola y Medicina Veterinaria presentan mayor deficiencia en ortografía, y las carreras de Administración Pública, Administración de Empresas, Medio Ambiente y Agroindustria reflejan menor cantidad de alumnado con deficiencia. En consecuencia, se comprueba que llegan a sus estudios universitarios con serias dificultades ortográficas y de terminología debido a que arrastran problemas adquiridos desde los niveles básicos y bachillerato.

En la dimensión de ortografía se identifican, con mayor frecuencia, la carencia de tildes, el uso erróneo de la grafías (b/v;s/c/z;h;g/j), la omisión o cambio de sílabas, errores que revelan el bajo desarrollo de habilidades lectoras y de escritura, lo cual refleja que no ha sido tratado por el profesorado con acciones metodológicas que ayuden a superar de manera eficiente los errores ortográficos del alumnado.

En lo que respecta al análisis de vocabulario, entre la escala cualitativa de deficiente y regular, la carrera de Medicina Veterinaria presenta el mayor porcentaje, seguida de Agrícola, Computación y Turismo, datos que coinciden con la problemática de las competencias ortográficas, pese a que, de manera global, el estudiantado de primer semestre de esta universidad se ubica en el nivel regular porque la falta de hábitos de lectura es evidente, pues la lectura es un factor que determina, en gran medida, la calidad de los conocimientos y desempeño del alumnado en formación profesionalizante.

Este estudio genera importante información sobre la realidad de la calidad educativa nacional y se convierte en un punto de partida para que la universidad asuma su rol protagónico y así, oriente una retroalimentación en el desarrollo de la ortografía y vocabulario que el estudiantado requiere para la superación de los problemas de escritura que presenta. Es urgente la intervención, a través de talleres de lectura y escritura, con reducido número de participantes y un plan de lectura coordinado con la biblioteca institucional.

\section{Referencias}

Camps, Anna., Milian, Marta., Bigas, Montserrat., Camps, Montserrat. y Cabré, Pilar. (1990). La enseñanza de la ortografía. Madrid, España: Editorial Graó.

Carlino, Paula. (2005). Escribir, leer y aprender en la universidad. Una introducción a la alfabetización académica. Buenos Aires, Argentina: Fondo de Cultura Económica. 
Carlino, Paula. (2013). Alfabetización académica diez años después. Revista mexicana de investigación educativa, 18(57), 355-381. Recuperado de https://www.redalyc.org/pdf/140/14025774003.pdf

Castelló, Montserrat. (2009). Aprender a escribir textos académicos: ¿Copistas, escribas, compiladores o escritores? En J.I. Pozo y M.P. Pérez (Coords.), Psicología del aprendizaje universitario: la formación en competencias (pp. 120-133). España: Ediciones Morata.

Castro, María. (2011). ¿Qué sabemos de la medida de las competencias? Características y problemas psicométricos en la evaluación de competencias. Bordón, 63(1), 109-123. Recuperado de https://dialnet.unirioja.es/descarga/articulo/3601046.pdf

Cisneros, Mireya, Muñoz, Clarena y Herrera, José. (2014). Perspectivas y prospectiva en los estudios sobre Lectura y Escritura. Risaralda, Colombia: Editorial Universidad Tecnológica de Pereira.

Condemarín, Mabel. y Medina, Alejandra. (2000). Evaluación de los aprendizajes. Un medio para mejorar las competencias lingüísticas y comunicativas. Santiago de Chile: MINEDUC.

Dido, Juan Carlos. (2001). Clínica de Ortografía: Una búsqueda abierta a nuevos recursos. Distrito Federal, México: Ediciones Novedades Educativas.

Errázuriz, María y Fuentes, Liliana. (2012). Diseño, implementación y evaluación de una propuesta de intervención en alfabetización académica en primer año de Pedagogía General Básica en la sede Villarrica de la UC. Onomázein: Revista de Lingüística, Filología y Traducción de la Pontificia Universidad Católica de Chile, 25(1), 287-313. Recuperado de https://dialnet.unirioja.es/servlet/articulo?codigo=3982914

Escarpanter, José. (1992). Introducción a la moderna gramática española. Colombia: Grupo Editorial Norma.

Franco, Ana y Benites, Lucrecia. (2018). Reflexiones acerca del currículo en la educación superior. Mikarimin: Revista Científica Multidisciplinaria, 4(4), 121-128. Recuperado de http://45.238.216.13/ojs/index.php/mikarimin/article/view/1192/411

Fernández-Rufete, Ana. (2015). Enseñanza de la ortografía, tratamiento didáctico y consideraciones de los docentes de Educación Primaria de la provincia de Almería. Revista Investigaciones sobre Lectura, (4), 7-24. Recuperado de https://dialnet.unirioja.es/descarga/articulo/5155197.pdf.

Ferreiro, Emilia. (1986). El proceso de alfabetización: La alfabetización en proceso. Buenos Aires, Argentina: Centro Editor de América Latina.

Flotts, Paulina., Manzi, Jorge., Jiménez, Daniela., Abarzúa, Andrea., Cayuman, Carlos. y García, María José. (2015). Logros de aprendizaje. Laboratorio Latinoamericano de Evaluación de la Calidad de la Educación (p. 153). [Informe de resultados TERCE, UNESCO, OREALC]. Recuperado de https://unesdoc.unesco.org/ark:/48223/pf0000243532 
Flotts, Paulina., Manzi, Jorge., Lobato, Patricia. Durán, María., Díaz, María. y Abarzúa, Andrea. (2016). Aportes para la enseñanza de la escritura. [Informe de resultados TERCE, UNESCO, OREALC]. Recuperado de https://unesdoc.unesco.org/ark:/48223/pf0000244734

Fonseca, Socorro, Correa, Alicia, Pineda, María y Lemus francisco. (2011). Comunicación oral y escrita. México: Pearson. Recuperado de https://bit.ly/3pcwW9x

García, Mari Paz. (2014). La evaluación de competencias en Educación Superior mediante rúbricas: un caso práctico. Revista Electrónica Interuniversitaria de Formación del Profesorado, 17(1), 87-106. doi: https://doi.org/10.6018/reifop.17.1.198861

Gómez Palacio, Margarita. (1998). Talleres de Escritura. México: SEP, PRONALEES.

Gómez Camacho, Alejandro. (2013). La competencia ortográfica en el alumnado de Ciencias de la Educación. En III Jornadas de Innovación Docente. Innovación Educativa: respuesta en tiempos de incertidumbre. Universidad de Sevilla, España. Recuperado de http://hdl.handle.net/11441/58873

González, Anielka, Rodríguez, Maylín y Ledo, Maritza. (2019). Enseñar a escribir en inglés: el enfoque proceso-producto orientado a la acción. Revista Transformación, 15(1), 14-26. Recuperado de http://scielo.sld.cu/scielo.php?script=sci arttext\&pid=S2077$\underline{29552019000100014}$

Guzmán, Natalia y Moya, María. (2016). Aplicación del programa "Buena Ortografía sin Esfuerzo" para mejorar el nivel ortográfico en el alumnado del I ciclo de la Escuela de Enfermería de la Universidad Católica Los Ángeles de Chimbote, 2010. Revista In Crescendo l, 7(1), 90-100. Recuperado de http://revistas.uladech.edu.pe/index.php/increscendo/issue/download/49/97

Hermosillo, Perla. y Verdín, Paulo. (2019). Perspectivas de la escritura académica en la universidad: causas, problemáticas y propuestas. Cuaderno de Pedagogía Universitaria, 16(31), 41-57. doi: https://doi.org/10.29197/cpu.v16i31.324

Kaufman, Ana María. (2017) ¿Es posible enseñar ortografía desde una perspectiva constructivista? Folios, (7), 22-32. https://doi.org/10.17227/01234870.7folios22.32

Murillo, Marielos. (2006). Vocabulario cacográfico. Pautas para la enseñanza de la ortografía en la escuela primaria costarricense. Káñina, 30(1), 59-70. Recuperado de https://revistas.ucr.ac.cr/index.php/kanina/article/viewFile/4640/4454

Namorado, Ana. (1995). El problema de lectoescritura en el estudiante universitario una perspectiva docente (Tesis de maestría). Universidad Autónoma de Nuevo León, México. Recuperado de http://eprints.uanl.mx/7133/1/1020091163.PDF

Ortiz, Marielsa., López, Trinidad., García, Marilín., Molina, Gisela., Pernía, Emiliana., Busto, Ivette., Rosales, Dianela., y Araujo, Élida. (2009). Representaciones y prácticas de docentes de educación básica en el campo de la lectura y la escritura. Letras, 51(79), 89-126. Recuperado de http://ve.scielo.org/pdf///v51n79/art04.pdf 
Palma, Delmis. (2012). Uso de estrategias didácticas para la enseñanza de la ortografía (escritura de palabras) a partir de situaciones comunicativas concretas, en el cuarto grado de la Escuela Primaria de Aplicación Musical de San Pedro Sula (Tesis de maestría). Universidad Pedagógica Nacional Francisco Morazán, Honduras. Recuperado de http://www.cervantesvirtual.com/nd/ark:/59851/bmc9p4s7

Pérez, Pilar y Zayas, Felipe. (2007). Competencia en comunicación lingüística. Madrid, España: Alianza Editorial.

Pérez, Sara, Federico, Navarro y Waigandt, Diana. (2017). Escribir para aprender y comunicar. Seminario de escritura académica. Argentina: Universidad Nacional de entre Ríos.

Prado, Josefina. (1998). Didáctica de la lengua y la literatura para educar en el siglo XXI (2a ed.). Madrid, España: La Muralla.

Prado, Josefina. (2016). Didáctica de la lengua y la literatura para educar en el siglo XXI (3a ed.). Madrid, España: La Muralla.

Real Academia Española. (1970). Diccionario de la lengua española (19a ed.). Madrid: Editorial Espasa Calpe

Ruiz, Maite. (2009). Evaluación de la lengua escrita y dependencia de lo literal. Barcelona, España: Graó.

Sánchez, David. (2009). Una aproximación a la didáctica de la ortografía en las clases de ELE. marcoele: Revista de didáctica Español como lengua extranjera, (9), 1885-2211. Recuperado de https://marcoele.com/descargas/9/sanchez ortografia.pdf.

Scardamalia, Marlene y Bereiter, Carl. (1992). Dos modelos explicativos de los procesos de composición escrita. Infancia y Aprendizaje, 58(15), 43-64. Recuperado de https://dialnet.unirioja.es/descarga/articulo/48395.pdf

Serrano de Moreno, María, Duque de Duque, Yolimar y Madrid de Forero, Alix. (2012). Prácticas de escritura académica en la universidad: ¿reproducir o transformar? Educare: Revista Venezolana de Educación, 16(53), 93-108. Recuperado de https://dialnet.unirioja.es/servlet/articulo?codigo $=6375676$

Thaiss, Chris y Zawacki, Terry. (2006). Engaged writers and dynamic disciplines: Research on the academic writing life. Portsmouth, New Hampshire: Boynton. Recovered from https://bit.ly/3p3owB9

Van Dijk, Teun. (2010). El estudio del discurso. En Discurso como estructura y proceso: estudios sobre el discurso. Introducción multidisciplinaria. México: Gedisa.

Vallejo, Raúl., Vidal, Gloria., Rivadeneira, Guido., Benavídez, Verónica. y Raza, Gladys. (2008). SER Sistema Nacional de Evaluación y Rendición Social de Cuentas. [Informe del Ministerio de Educación, SER Ecuador]. Recuperado de https://educacion.gob.ec/wpcontent/uploads/downloads/2013/04/SIST NAC EVALUACION FF.pdf 
Revista indizada en

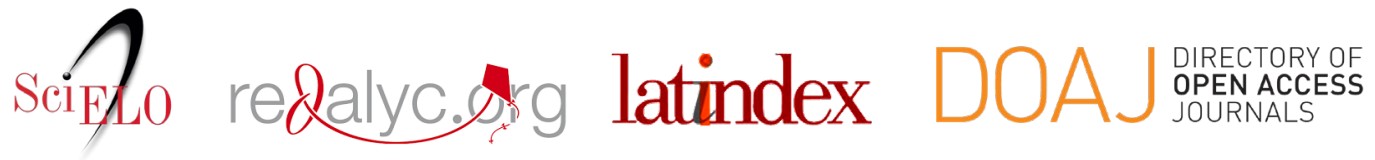

Distribuida en las bases de datos:

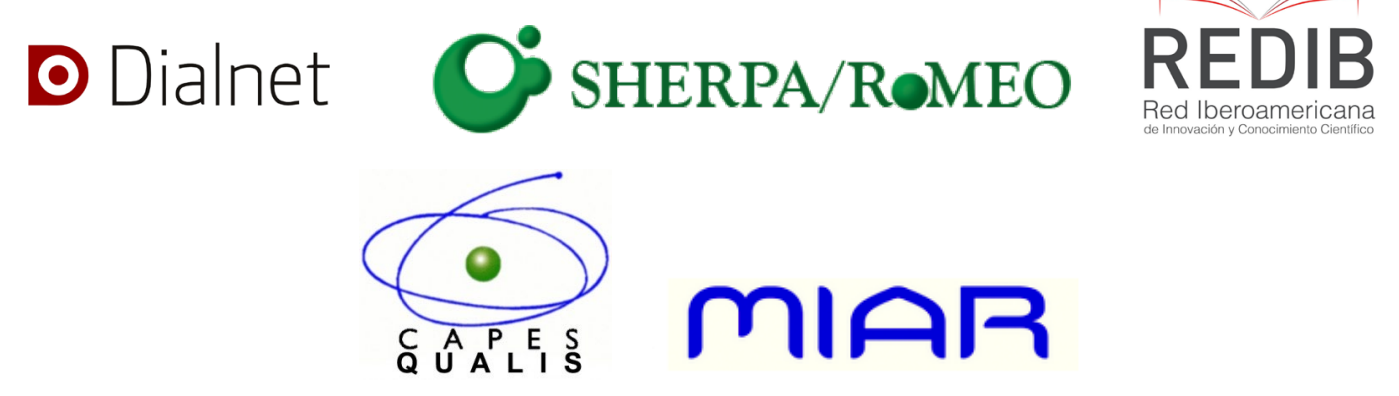

